\section{Zur Kenntnis des Molekulargewichtes von Peptidsynthetasen aus Leber und Hefe}

J. Dimigen, F. Klink und D. Richter

Institut für physiologische Chemie und Physikochemie der Universität Kiel

(Z. Naturforschg. 20 b, 924-925 [1965] ; eingegangen am 23. Juli 1965)

Die polysomale Peptidsynthese wird durch mindestens zwei verschiedene Enzyme katalysiert. Aus $E$. $\operatorname{coli}^{1}$, Reticulocyten ${ }^{2}$, Leber ${ }^{3-5}$ und Hefe ${ }^{6}$ wurden bisher je ein sehr labiler und ein wesentlich weniger empfindlicher Transferfaktor angereichert. Bei Reticulocyten ${ }^{2}$ und Leber ${ }^{7}$ scheint der labile Faktor die Anlagerung von tRNS ${ }^{11}$ an Ribosomen zu beeinflussen, so daß man dem stabileren Enzym die unmittelbar Peptid-knüpfende Wirkung zuschreiben darf. Keiner dieser Faktoren wurde bisher soweit gereinigt, da $\beta$ eine Bestimmung physikochemischer Konstanten möglich war. Gelfiltration an Dextrangelen erlaubt jedoch bei vielen globulären Proteinen eine recht genaue Abschätzung der Molekulargröße auch ohne Reindarstellung ${ }^{8,9}$. Wir wendeten diese Methode auf stark angereicherte Präparationen des stabileren Faktors (F I) aus Leber und Hefe an.

\section{Methoden}

Zur Abtrennung des Enzyms von dem labileren Faktor (F II) und zur weiteren Anreicherung verwendeten wir neben den früher beschriebenen chromatographischen Verfahren mit TEAE-Zellulose ${ }^{5}$ mehrere Ammoniumsulfat-Fraktionierungsschritte und Gelfiltration an Sephadex G 25 und G $200^{7}$.

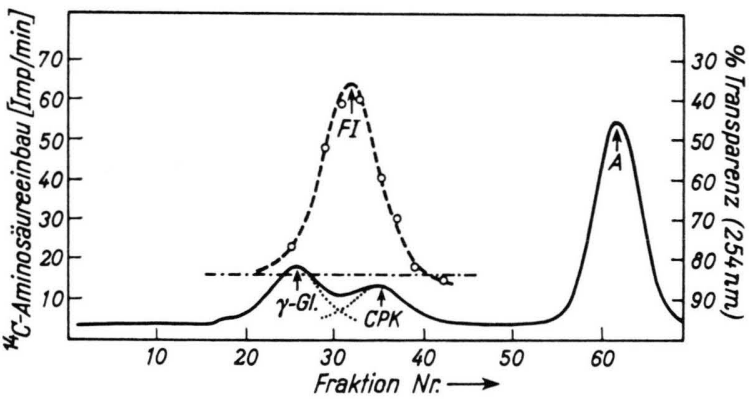

Abb. 1. Mit Standardproteinen gewonnene Eichkurve zur Bestimmung von Mol.-Gew. durch Gelfiltration an Sephadex G 200. Das durchbrochene Kästchen gibt den Bereich, in dem die für FI aus Ratten- und Kalbsleber in verschiedenen Experimenten ermittelten Werte liegen.

1 J. E. Allende, R. Monro u. F. Lipman, Proc. nat. Acad. Sci. USA 51, 1211 [1964].

2 R. Arlinghaus, J. Shaeffer u. R. Schweet, Proc. nat. Acad. Sci. USA 51, 1291 [1964].

3 J. M. Fessenden u. K. Moldave, J. biol. Chemistry 238, 1479 [1963].

4 S. Slapikoff, J. M. Fessenden u. K. Moldaye, J. biol. Chemistry 238, 3670 [1963].

\begin{tabular}{|c|c|c|c|}
\hline Protein & Reinheitsgrad & Bezugsquelle & $\begin{array}{l}\text { Mol.- } \\
\text { Gew. }\end{array}$ \\
\hline $\begin{array}{l}\gamma \text {-Globulin } \\
\text { (Rind) } \\
\text { Serumalbumin, } \\
\text { dimer (Rind) } \\
\text { Creatinphospho- } \\
\text { kinase } \\
\text { Serumalbumin } \\
\text { (Rind) } \\
\text { Eieralbumin } \\
\text { Rennin }\end{array}$ & $\begin{array}{l}\text { trocken, reinst } \\
\text { trocken, reinst } \\
\text { p. a. } \\
\text { trocken, reinst } \\
\text { 5-mal krist., } \\
\text { reinst } \\
\text { Krist. }\end{array}$ & $\begin{array}{l}\text { Behring } \\
\text { (Marburg) } \\
\text { Behring } \\
\text { (Marburg) } \\
\text { Boehringer } \\
\text { (Mannheim) } \\
\text { Behring } \\
\text { (Marburg) } \\
\text { Serva } \\
\text { (Heidelberg) } \\
\text { Nutr. Bioch. } \\
\text { Corp., } \\
\text { Cleveland, } \\
\text { Ohio }\end{array}$ & $\begin{array}{r}160000 \\
134000 \\
81000 \\
67000 \\
45000 \\
34500\end{array}$ \\
\hline
\end{tabular}

Tab. 1. Standardproteine mit Mol.-Gew. (Literaturwerte), die zur Eichung der Sephadexsäulen verwendet wurden.

Die Aktivitätsbestimmung von F I wurde durch Inkubation mit Polysomen aus Leber, ${ }^{14} \mathrm{C}$-AminoacyltRNS, den notwendigen Cofaktoren und Ionen sowie einem Überschuß von F II durchgeführt (Einzelheiten s. Abb. 2).

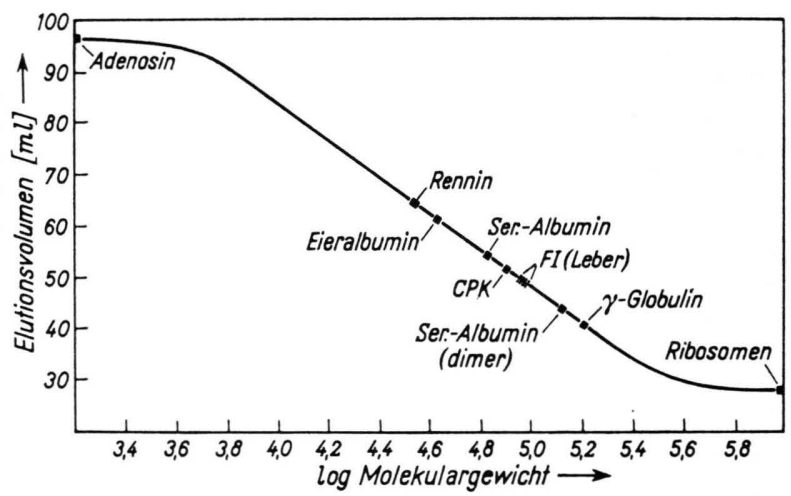

Abb. 2. Trennung von FI aus Rattenleber, CPK, $\gamma$-Globulin und Adenosin an Sephadex G 200. Transparenz - ; Transferenzymaktivität $0_{---}$. Das Testsystem ${ }^{11}$ enthielt in $1 \mathrm{ml}: 8 \mu \mathrm{Mol} \mathrm{Mg}\left(\mathrm{CH}_{3} \mathrm{COO}\right)_{2} ; 100 \mu \mathrm{Mol} \mathrm{KCl} ; 50 \mu \mathrm{Mol}$ Tris $\left(p_{\mathrm{H}} 7,6\right) ; 30 \mu \mathrm{Mol}$ MEA; $2 \mu \mathrm{Mol}$ ATP; $0,33 \mu \mathrm{Mol}$ GTP; $4 \mu$ Mol PEP; $25 \mu \mathrm{g}$ PK; 5,0 Ext. Einh. $(260 \mathrm{~nm}) /$ $1 \mathrm{~cm}$ Rattenleber-Polysomen; $50 \mu \mathrm{g}{ }^{14} \mathrm{C}$-Aminoacyl-tRNS $=3000 \mathrm{Ipm} / \mathrm{min} ; 0,2 \mathrm{ml}$ FII aus Hefe; $0,3 \mathrm{ml}$ der bezeichneten Eluatfraktionen. Aufarbeitung der markierten Proteine durch TCE-Fällung, Waschung mit heißer TCE, Alkohol-Äther, Äther; Aktivitätsmessung im Methandurchflußzähler.

5 F. Klink, A. M. Nour u. K. F. Aepinus, Biochim. biophysica Acta [Amsterdam] 72, 654 [1963].

6 F. KLink u. D. Richter, in Vorbereitung.

7 G. Kramer, F. Klink u. H. G. Petersen, in Vorbereitung.

8 P. Andrews, Biochem. J. 91, 222 [1964].

9 F. Auricchio u. C. B. Bruni, Biochem. Z. 340, 321 [1964].

10 J. Bishop u. R. Schweet, Biochim. biophysica Acta [Amsterdam] 49, 235 [1961]. 
Gelfitration: Sephadex G 200 wurde 72 Stdn. in Puffer (0,05-m. Tris, 0,1-m. KCl, 0,008-m. $\mathrm{Mg}\left(\mathrm{CH}_{3} \mathrm{COO}\right)_{2}$, $\left.p_{\mathrm{H}} 7,6\right)$ gequollen. Die Säule (Vol. $90 \mathrm{ml} ; 550 \cdot 14 \mathrm{~mm}$ ) wurde nach dem Gießen 48 Stdn. mit Puffer durchströmt, das äußere Volumen mit Ribosomen oder Dextranblau, das innere mit Adenosin bestimmt, die Extinktion des Eluates im Durchflußphotometer bei $254 \mathrm{~nm}$ gemessen und die Lage der Gipfel durch zusätzliche Messung bei 280 bzw. $260 \mathrm{~nm}$ präzisiert. Zur Kalibrierung der Säulen wurden reine Proteine mit bekanntem Mol.-Gew. verwendet (s. Tab. 1). Bei logarithmischer Auftragung ${ }^{8}$ der Mol.-Gew. gegen das Elutionsvolumen (Abb. 1) ergab sich eine über weite Bereiche lineare Abhängigkeit.

\section{Ergebnisse}

Abb. 2 zeigt eine Auftrennung eines Gemisches von F I, Creatinphosphokinase (CPK), $\gamma$-Globulin und Adenosin an Sephadex G 200. Die Transfer-Enzymaktivität wurde in einem einheitlichen Gipfel eluiert. Die aktiven Fraktionen hatten in Abwesenheit von F II keine Wirkung im Testsystem. Die Elutionsvolumina für F I-Präparate aus Ratten- und Kalbsleber ergaben Mol.-Gew. zwischen 89000 und 95000 . Bei Anwesenheit von 0,005-m. MEA ${ }^{11}$ im Elutionsmedium zeigte sich keine Verschiebung des Aktivitätsgipfels. Ein Einfluß der für die Transferreaktion unentbehrlichen Sulfhydrylsubstanzen ${ }^{\mathbf{1 0}}$ auf die Molekulargröße (etwa durch Desaggregation) ist somit nicht feststellbar. Die deutlich geringeren Werte - etwa 80000 - die an F I aus Hefe gemessen wurden, bedürfen der Sicherung durch weitere Experimente.

Frl. I. LANGE sind wir für sorgfältige technische Mitarbeit, der Deut s chen Forschungsgemeins chaft für die Unterstützung dieser Arbeit zu Dank verpflichtet.

11 Abkürzungen: ATP $=$ Adenosintriphosphat. GTP = Guanosintriphosphat. $\mathrm{PEP}=$ Phosphoenolpyruvat. $\mathrm{PK}=$ Pyru vatkinase. tRNS $=$ Transfer-Ribonucleinsäure. TCE = Trichloressigsäure. MEA =2-Mercaptoaethylamin.

\section{Zur Abhängigkeit der photosynthetischen NADP-Reduktion von Plastocyanin}

\section{A. Trebst und E. Elstner}

Pflanzenphysiologisches Institut der Universität Göttingen, Abt. Biochemie der Pflanzen

(Z. Naturforschg. 20 b, 925-926 [1965] ; eingegangen am 3. August 1965)

Hinweise für eine Funktion des von $\mathrm{K}_{\mathrm{ATOH}}$ und $\mathrm{T}_{\mathrm{A}}$ камгуа isolierten Plastocyanins ${ }^{1}$ in der Photosynthese ergaben sich aus einer Reihe von Ergebnissen. Die photosynthetischen Reaktionen isolierter Chloroplasten wurden durch $\mathrm{Cu}$-Komplexbildner, insbesondere Salicylaldoxim ${ }^{2}$, gehemmt, die Cytochrom c-Photooxydation in Digitonin-fragmentierten, Plastocyanin-freien Chloroplasten ist Plastocyanin-abhängig ${ }^{3}$, die Absorption bei $600 \mathrm{~m} \mu$ (Absorptionsmaximum des Plastocyanins) ändert sich bei Belichtung der Algen unter verschiedenen Bedingungen ${ }^{4}$ und reduziertes Plastocyanin wird durch das Pigmentsystem 1 der Photosynthese oxy$\operatorname{diert}^{5}$. Diese Versuche stimmten darin überein, daß

1 S. КатоH, Nature [London] 186, 533 [1960]; S. КатоH u. A. Takamiya, Nature [London] 189, 665 [1961]; S. Katoh, I. Suga, I. Shiratori u. A. Takamiya, Arch. Biochem. Biophysics 94, 136 [1961].

2 A. Trebst, Z. Naturforschg. 18 b, 817 [1963]; A. Trebst, H. Eck u. S. WAGNeR, in: „Photosynthetic Mechanism of Green Plants“, National Academy of Sciences - National Research Council, Washington 1963.

3 S. КатоH u. A. Takamiya, in: „Photosynthetic Mechanisms of Green Plants“, National Academy of Sciences National Research Council, Washington 1963; S. КАтоH u. A. Takamiya, Plant Cell Physiol. 4, 335 [1963] .

4 J. de Kouchrovsky u. D. C. Fork, Proc. nat. Acad. Sci. USA 52, 232 [1964]; D. C. Fork u. W. Urbach, Proc. nat. Acad. Sci. USA 53, 1307 [1965].
Plastocyanin als „electron carrier“ zwischen den beiden Lichtreaktionen der Photosynthese einzuordnen ist.

Wir möchten hier über eine einfache Methode berichten, die Plastocyanin-Abhängigkeit der NADP-Reduktion zu zeigen. Sie baut auf den Versuchen von Nieman und Vennesland ${ }^{6}, \mathrm{~K}_{\mathrm{atoH}}{ }^{3}$ und $\mathrm{KoK}^{5}$ mit Detergenz-fragmentierten Chloroplasten auf. Solche Chloroplasten haben die Fähigkeit zur $\mathrm{O}_{2}$-Entwicklung verloren, können aber - im Gegensatz zu unbehandelten Chloroplasten - reduziertes Cytochrom c photooxydieren. Whatley ${ }^{7}$ zeigte, daß diese Cytochrom cOxydation fragmentierter Chloroplasten auch zur Reduktion von NADP verwendet werden kann, wenn Ferredoxin und Ferredoxin-NADP-Reduktase zugesetzt werden. Niemann und Vennesland ${ }^{6}$ fanden, daß ÄthanolFällung solcher fragmentierter Chloroplasten zu Partikeln führt, die Cytochrom c erst nach Zugabe eines Faktors aus dem Überstand photooxidierten, den $\mathrm{K}_{\mathrm{ATOH}}$ und TAkafiya ${ }^{3}$ als Plastocyanin identifizierten. Die Arbeiten von $\mathrm{KoK}^{5}$ zeigen deutlich, daß das Pigmentsystem 1 der Photosynthese nach Detergenz-Behandlung intakt geblieben ist und daß es Plastocyanin und Cytochrom $\mathrm{f}$ bei Belichtung oxydiert. Wir zeigten kürzlich ${ }^{8}$,

5 B. Koк, in: „Photosynthetic Mechanisms of Green Plants“, National Academy of Sciences - National Research Council, Washington 1963; B. Кок, H. J. Rurainski u. E. A. Harmon, Plant Physiol. 39, 513 [1964]; B. Koк u. H. J. Rurainski, Biochim. biophysica Acta [Amsterdam] 94, 588 [1965].

6 R. H. Nieman u. B. Vennesland, Plant Physiol. 34, 255 [1959]; R. H. Nieman, H. NaKamura u. B. Vennesland, Plant Physiol. 34, 262 [1959].

7 F. R. Whatley, in: „Photosynthetic Mechanisms of Green Plants", National Academy of Sciences - National Research Council, Washington 1963.

8 A. Trebst u. E. Pistorius, in: „Beiträge zur Biochemie und Physiologie von Naturstoffen", Festschrift zum 65. Geburtstag v. K. Мотнеs, VEB Fischer Verlag, Jena 1965. 